$52 / 30-96820$

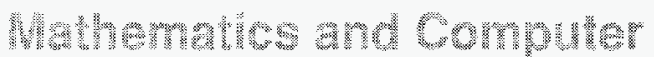

somitug bivision

ANL-96/5

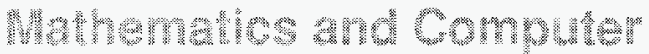

Sclence Divisum

WhHemalcs and Computer

solstuse oluminn

Wathematros and Compur

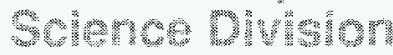

Mathematics and Computer Science Division Mathematics and Computer Science Division Mathematics and Computer Science Division Mathematics and Computer by W. Gropp and E. Lusk

Sclence Division Mathematios and Conputer

Science Dhision Mathematics and Computer

Sclence Division Mathematios and Computer

Science Division Mathenatics and Computer

Sclence Division Mathematics and Computer Science Division Mathemalcs and Computer

Science DViston Mathemalics and Computer

Science Division Installation Guide to mpich, a
Portable Implementation of MPI cs and Computer

Sclence Quiston Mathemallos and Comouter

Science Division

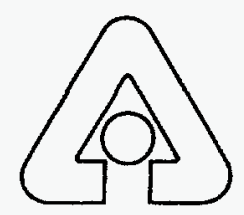

Argonne National Laboratory, Argonne, Illinois 60439

operated by The University of Chicago

for the United States Department of Energy under Contract W-31-109-Eng-38

Mathematics and Computer

Seience Division

Wathematcs and Computer

Science Division

Mathematics and Computer

Science Division

Mathematics and Computer

DISTRIBUTION OF THIS DOCUMENT IS UNLIMITEO 85

Sclence Division 
Argonne National Laboratory, with facilities in the states of Illinois and Idaho, is owned by the United States government, and operated by The University of Chicago under the provisions of a contract with the Department of Energy.

\section{DISCLAIMER}

This report was prepared as an account of work sponsored by an agency of the United States Government. Neither the United States Government nor any agency thereof, nor any of their employees, makes any warranty, express or implied, or assumes any legal liability or responsibility for the accuracy, completeness, or usefulness of any information, apparatus, product, or process disclosed, or represents that its use would not infringe privately owned rights. Reference herein to any specific commercial product, process, or service by trade name, trademark, manufacturer, or otherwise, does not necessarily constitute or imply its endorsement, recommendation, or favoring by the United States Government or any agency thereof. The views and opinions of authors expressed herein do not necessarily state or reflect those of the United States Government or any agency thereof.

Reproduced from the best available copy.

Available to DOE and DOE contractors from the

Office of Scientific and Technical Information

P.O. Box 62

Oak Ridge, TN 37831

Prices available from (423) 576-8401

Available to the public from the

National Technical Information Service

U.S. Department of Commerce

5285 Port Royal Road

Springfield, VA 22161 
ARGONNE NATIONAL LABORATORY

9700 South Cass Avenue

Argonne, IL 60439

ANL-96/5

\section{Installation Guide to mpich, a Portable Implementation of MPI}

by

William Gropp and Ewing Lusk

Mathematics and Computer Science Division

July 1996

This work was supported by the Mathematical, Information, and Computational Sciences Division subprogram of the Office of Computational and Technology Research, U.S. Department of Energy, under Contract W-31-109-Eng-38. 


\section{DISCLAIMER}

Portions of this document may be illegible in electronic image products. Images are produced from the best available original document. 


\section{Contents}

$\begin{array}{ll}\text { Abstract } & 1\end{array}$

1 Quick Start 1

2 Obtaining and Unpacking the Distribution 2

3 Documentation 4

4 Configuring mpich 4

4.1 Building a Production mpich . . . . . . . . . . . . . 12

4.2 What If There Is No Fortran Compiler? . . . . . . . . . . . . . 12

4.3 Special Issues for Heterogeneous Networks . . . . . . . . . . . . . . 12

5 Compiling mpich $\quad 13$

5.1 Getting tcl, tk, and wish . . . . . . . . . . . . 13

5.2 Building Multiple Devices or Architectures f............ 13

6 Running an MPI Program $\quad 13$

6.1 Special Considerations for Running on a Network of Workstations . . . . 15

6.1 .1 Dealing with Automounters .................. 15

6.1 .2 Faster Job Startup . . . . . . . . . . . . . . . 16

6.1 .3 Stopping the Servers .......................... 18

6.1 .4 Managing the Servers ...................... 18

6.2 Special Considerations for Running with Shared Memory . . . . . . . 19

7 Thorough Testing $\quad 19$

8 Installing mpich for Others to Use $\quad 20$

8.1 User Commands ......................... 20

8.2 Installing Documentation . . . . . . . . . . . . . 21

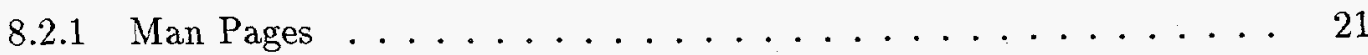

8.2 .2 Web Versions of Man Pages . . . . . . . . . . . . . 21

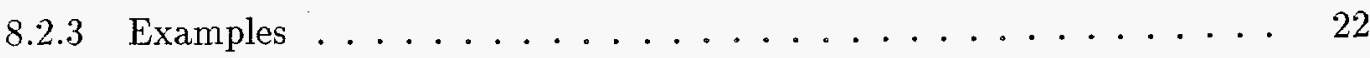


10 Benchmarking mpich

11 The mpich Programming Environment 23

11.1 Introduction . . . . . . . . . . . . . . . . 23

11.2 mpirun, a Portable Startup Script . . . . . . . . . . . . . 24

11.3 The mpicc and mpif77 Commands . . . . . . . . . . . . 28

11.4 mpireconfig, a Way to Create Makefiles . . . . . . . . . . . 29

11.5 nupshot, a Way to View Logfiles . . . . . . . . . . . . . . . 29

12 Automatic Report Generation $\quad 29$

13 Problems $\quad 30$

13.1 Submitting Bug Reports . . . . . . . . . . . . . . . 30

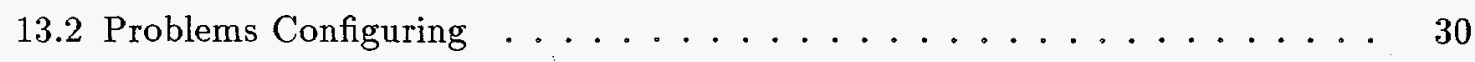

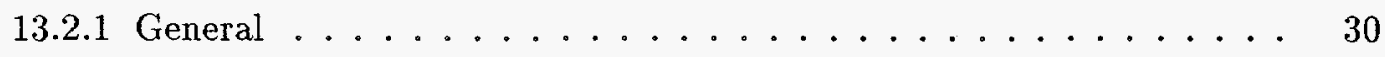

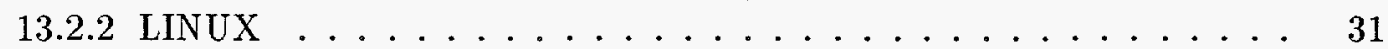

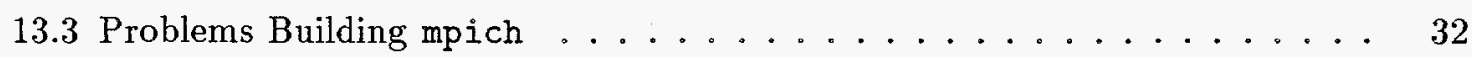

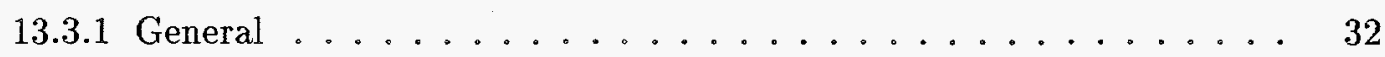

13.3.2 Workstation Networks ................. 33

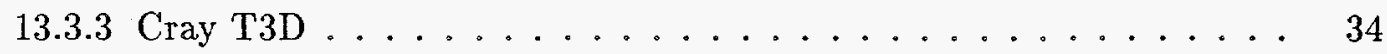

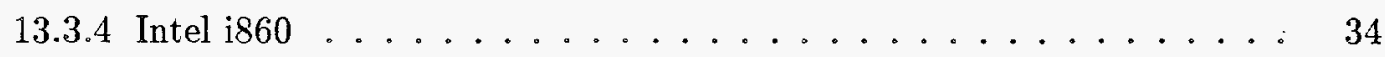

13.3.5 Intel Paragon . . . . . . . . . . . . . . . . 34

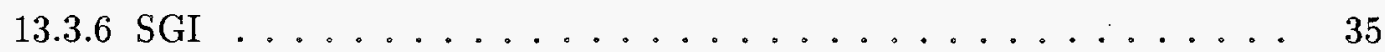

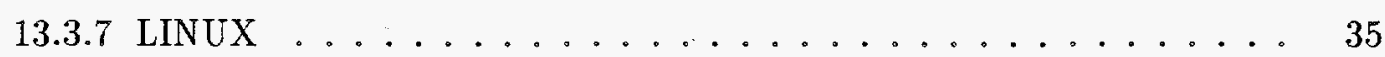

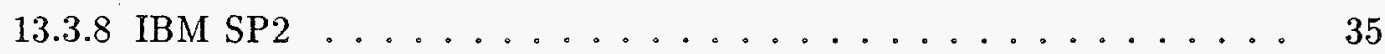

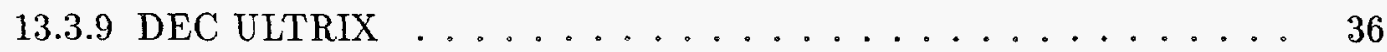

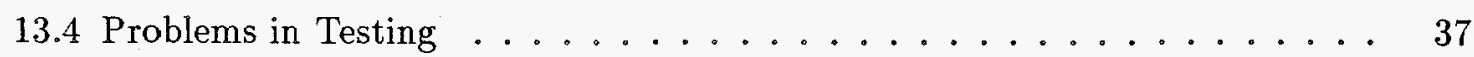

$\begin{array}{ll}\text { Acknowledgments } & 37\end{array}$

$\begin{array}{ll}\text { References } & 38\end{array}$ 


\title{
Installation Guide to mpich, a Portable Implementation of MPI
}

by

\author{
William Gropp and Ewing Lusk
}

\begin{abstract}
MPI (Message Passing Interface) is a standard specification for message-passing libraries. mpich is a portable implementation of the full MPI specification for a wide variety of parallel computing environments, including workstation clusters and massively parallel processors. mpich contains, along with the MPI library itself, a programming environment for working with MPI programs. The programming environment includes a portable startup mechanism, several profiling libraries for studying the performance of MPI programs, and an X interface to all of the tools. This guide explains how to compile, test, and install mpich and its related tools.
\end{abstract}

This is a document in progress: hopefully helpful, but hardly whole. Please send suggestions for improvements (or impairments) to mpi-bugs@mcs.anl.gov. Details on using the MPICH implementation are presented in a separate users guide for mpich.

\section{Quick Start}

Here is a set of steps for setting up and minimally testing mpich. Details and instructions for a more thorough tour of mpich's features, including installing, validating, benchmarking, and using the performance evaluation tools, are given in the following sections.

1. If you have gunzip, get mpich.tar.gz; otherwise, get mpich.tar.Z by anonymous ftp from info.mcs.anl.gov in the directory pub/mpi. (If that file is too big, try getting the pieces from pub/mpi/mpisplit and cating them together.)

2. gunzip -c mpich.tar | tar xovf - or zcat mpich.tar.z | tar xovf -

3. cd mpich

4. configure - $\operatorname{arch}=\operatorname{sun} 4-$ device $=c h \_p 4$ (for example)

5. make >\& make.log (in C-shell syntax). Depending on the load on your system and on your file server, this may take anywhere from 10 minutes to an hour or more.

6. To run workstation networks or on a single workstation, edit the file mpich/util/machines/machines.sample to reflect your local host names for the machines of the architecture given with - arch= above. On parallel machines, this step is not needed. See the README file in the mpich/util/machines directory for a description of the format.

7. Build and run a simple test program: 
cd examples/basic

make cpi

mpirun -np 4 cpi

8. make PREFIX=/usr/local/mpi install to install MPICH into /usr/local/mpi.

9. (Optional) Build the rest of the mpich environment: For the ch_p4 device, use of the secure server (see Section 6.1.2) can speed job startup; you can build it with

make serv_p4

The nupshot program is a faster version of upshot, but requires tk source code. If you have this package, you can build nupshot with

make nupshot

At this point you have run an MPI program on your system. In the following sections we go through these steps in more detail and describe other aspects of the mpich distribution you might wish to explore.

The companion users guide [5] gives more information on building and running MPI programs with mpich.

\section{Obtaining and Unpacking the Distribution}

mpich can be obtained by anonymous ftp from the site info.mcs.anl.gov. Go to the directory pub/mpi and get the file mpich.tar.z. This file name is a link to the most recent verstion of mpich. Currently it is about 4 megabytes in size. The file is a compressed tar file, so it may unpacked with

zcat mpich.tar.z I tar xvf -

If your system does not have zcat, you must uncompress it in a separate step:

uncompress mpich.tar.Z

tar xvf mpich.tar

This will create a single directory called mpich, containing in various subdirectories the entire distribution, including all of the source code, some documentation (including this guide), man pages, the mpich environment described in Section 11, and example programs. In particular, you should see the following files and directories:

COPYRIGHT Copyright statement. This code is free but not public domain. It is copyrighted by the University of Chicago and Mississippi State University.

Makefile.in Template for the Makefile, which will be produced when you run configure.

README Basic information and instructions for configuring. 
aclocal.m4 Used for building configure from configure.in; not needed for most installations.

bin Home for executable files like mpirun and mpiman.

ccbugs Directory for programs that test the $\mathrm{C}$ compiler during configuration, to make sure that it will be able to compile the system.

configure The script that you run to create Makefiles throughout the system.

configure.in Input to autoconf that produces configure.

doc Assorted documentation, including this guide.

examples Directory containing further directories of example MPI programs. Of particular note are basic, with a few small examples to try first, test, with a test suite for exercising mpich, and perftest, containing benchmarking code.

include The include libraries, both user and system.

installtest A place to test the installation script.

lib The machine-dependent libraries, after they are built. Subdirectories are maintained for each version of the system that is built, so this same tree can be host for multiple installations. Machine-dependent scripts such as mpirun and mpireconfig are also kept here.

man Man pages for MPI, MPE, and internal routines.

mpe The source code for the MPE extensions for logging and X graphics. The contrib directory contains examples. Best are the mandel and mastermind subdirectories.

mpid The source code for the various "devices" that customize mpich for a particular machine, operating system, and environment.

profiling The profiling subsystem, including a system for automatically generating the "wrappers" for the MPI profiling interface.

ptx_ifile A file needed by the Sequent Symmetry running the PTX operating system.

ref Postscript versions of the reference manuals.

src The source code for the portable part of mpich. There are subdirectories for the various parts of the MPI specification.

util Utility programs and files.

If you have problems, check the MPICH home page on the Web at http://www.mcs.anl.gov/Projects/mpi/mpich. This page has pointers to lists of known bugs and patchfiles. If you don't find what you need here, send mail to mpi-bugs@mcs . anl.gov. 


\section{Documentation}

This distribution of mpich comes with complete man pages for the MPI routines, the MPE extensions, and the ADI (Abstract Device Interface) routines. The command mpiman in mpich/bin is a good interface to the man pages. ${ }^{1}$ The mpich/ref directory contains printable versions of the manuals for MPI and the ADI, in compressed PostScript form.

\section{Configuring mpich}

The next step is to configure mpich for your particular computing environment. mpich can be built for a variety of parallel computers and also for networks of workstations. Parallel computers supported include the IBM SP1 and SP2 (using various communication options), the TMC CM-5, the Intel Paragon, IPSC860, and Touchstone Delta, the Ncube Ncube2, the Meiko CS-2, the Kendall Square KSR-1 and KSR-2, Convex Exemplar, and IBM, SGI and Sun multiprocessors. Workstations supported are the Sun4 family, Hewlett-Packard, DEC 3000 and Alpha, IBM RS/6000 family, and SGI. Also supported are Intel x86-based PC clones running the LINUX or FreeBSD operating systems. New ports are always pending.

Configuration of mpich is done with the configure script contained in the top-level directory. This script is automatically generated by the Gnu autoconf program from the file configure. in, but you do not need to have autoconf yourself.

The configure script documents itself in the following way. If you type

$$
\text { configure -usage }
$$

you will get the following.

Configuring with args -usage

Configuring MPICH Version 1.0.13.

Usage: /home/MPI/mpich/configure -arch=ARCH_TYPE -comm=COMM_TYPE -device=DEVICE

[-prefix $=$ INSTALL_DIR $] \quad[-\mathrm{C}++[=\mathrm{C}++$-COMPILER $]]$

$\left[-\mathrm{CC}=\mathrm{C}_{-}\right.$COMPILER] [-fC=FORTRAN_COMPILER]

[-clinker $=$ C_LINKER $][-$ flinker $=$ FORTRAN_IINKER]

[-mpe] [-nompe] [-nof77] [-f90nag] [-opt=OPTFLAGS]

[-make=MAKEPGM]

[-cflags $=$ CFLAGS $][$-fflags $=$ FFLAGS $]$

[-optcc $=$ C_OPTFLAGS] [-opt $f 77=$ F77_OPTFLAGS $]$

[-lib=LIBRARY] [-mpilibname=MPINAME]

[-no_mpegraphics] [-no_short_longs] [-memdebug]

$\left[-\mathrm{x} 11_{-} \mathrm{lib}=\mathrm{X} 11 \mathrm{LIB}\right] \quad\left[-\mathrm{x} 11_{-}\right.$inc $\left.=\mathrm{X} 11 \mathrm{INC}\right]$

[-mpedbg] [-nompedbg] [-cross] [-devdebug] [-nodevdebug]

[-debug] [-nodebug]

[-var_pkt] [-pkt_size=LENGTH] [-adi_collective]

[-adi_coll_world]

[-wish=WISH $][-t c l d i r=T C L D I R] \quad[-t k d i r=T K D I R]$

\footnotetext{
${ }^{1}$ The mpiman command is created by the configure process described later.
} 


\section{[-fortnames=FORTRANNAMES] \\ [-ar_nolocal] [-automountfix=AUTOMOUNTFIX] \\ [-noranlib] [-rsh=RSHCOMMAND] [-rshnol]}

where

ARCH_TYPE = the type of machine that MPI is to be configured for

COMM_TYPE = communications layer or option to be used

DEVICE = communications device to be used

INSTALL_DIR = directory where MPI will be installed (optional)

C++_COMPILER = default is to use $\mathrm{g++}$ (optional)

OPTFLAGS = optimization flags to give the compilers (e.g. $-\mathrm{g})$

CFLAGS $\quad$ flags to give $C$ compiler

FFLAGS $\quad=$ flags to give Fortran compiler

MAKEPGM = version of make to use

LENGTH = Length of message at which ADI switches from short to long message protocol

WISH $=$ Name of $t c l / t k$ wish executable. Configure will attempt to find a version of wish for you, but if there is no wish in your path or you need to use a different version, use this option. Used only for the display tools (nupshot and upshot). tk $3 . x$ required for nupshot; tk $3 . x$ or $4 . x$ for upshot.

TCLDIR = Directory containing tcl. Must have lib/libtcl.a and include/tcl.h. Used only for nupshot.

TKDIR = Directory containing tk $3.3,3.4,3.5$, or 3.6 . Must have lib/libtk.a and include/tk.h. Used only for nupshot. May be the same as TCLDIR.

FORTRANNAMES $=$ Form of the Fortran names. See below.

X11LIB $\quad$ Full path name for IibX11.a

$\mathrm{X} 11 \mathrm{INC} \quad=$ Full path name for X11.h

AUTOMOUNTFIX $=$ Command to $\mathrm{f} i \mathrm{x}$ automounters

RSHCOMMAND = Command to use for remote shell

MPILIBNAME = Name to use instead of mpi in the name of the MPI library. If set, libMPILIBNAME will be used instead or libmpi. This can be used on systems with several different MPI implementations.

One and only one 'arch', 'comm', and 'prefix' argument should be provided. 'arch' MUST be specified before 'comm'.

If '-ct+' is included as an option, then the C++ interface is also built. By default, $\mathrm{g}^{++}$is used as the c++ compiler. THIS IS CURRENTLY UNSUPPORTED.

You can select a different $C$ and Fortran compiler by using the '-cc' and ' $f c$ ' switches. The environment variables ' $\mathrm{CC}$ ' and ' $\mathrm{FC}$ ' can also provide values for these but their settings may be overridden by the configure script. Using ' $-c c=\$ C C-f C=\$ F C$ ' will force configure to use those compilers.

If '-cross' is given, configure assumes that you are cross-compiling. If it 
is not given, configure expects to be able to run programs. Even if '-cross' is not selected, configure will try to determine if you are cross-compiling; this switch is needed only on systems where attempting to run a cross-compiled program causes the configure script to hang.

If '-mpe' is included as an option, then the MPE 'helper' libraries will also be built. '-nompe' causes the MPE libraries to not be built. The default is '-mpe'. If '-no_mpegraphics' is used, then the MPE routines that make use of X11 graphics will NOT be built; this is appropriate for systems that do not have the $X 11$ include files or that do not support $X 11$ graphics (some message-passing systems cannot interoperate with $\mathrm{X} 11$ ). The options $-\mathrm{x} 11$ inc and -x11_lib may be used to specify the locations of the X11 include files and libraries in the event that configure cannot find them (they should both be specified in that case).

The option '-mpedbg' enables the '-mpedbg' command line switch in MPI programs. When used with an MPI program, the default error handler (i.e., MPI_COMM_WORLD's error handler) tries to start xterm's running $d b x$ for each process that detects an error. This option is intended primarily for workstation environments but should work on some MPPs (such as IBM SP2).

The option '-nof 77' prevents the compilation of routines that require a Fortran compiler. If this option is selected, you may not use the Fortran interface to MPI.

The option '-f90nag' allows you to use the NAG Fortran 90 instead of Fortran 77. This is a preliminary version and is based on the version for NeXTs.

The option '-opt' allows you to specify options for the compilers (both $C$ and Fortran). For example, '-opt $=-0$ ' chooses optimized code generation on many systems. '-optcc' and '-optf77' allow you to specify options for just the C or Fortran compilers

The option '-lib' allows you to specify the location of a library that may be needed by a particular device. Most devices do NOT need this option; check the installation instructions for those that might.

The option '-make' may be used to select an alternate make program. For example, on FreeBSD systems, -make=gnumake may be required because of bugs in the system make.

The option '-no_short_longs' may be used to suppress support for ANSI C types 'long long int' and 'long double' when they are the same size as 'long' and 'double' respectively. Some systems allow these long ANSI C types, but generate a warning message when they are used; this option may be used to suppress these messages (and support for these types).

The option '-fortnames=FORTRANNAMES' allows you to specify the form of the 
Fortran names. This is used primarily to generate names with and without trailing underscores for those systems that support both. Possible values are FORTRANNAMES value if Fortran MPI_SEND looks like DOUBLEUNDERSCORE UNDERSCORE CAPS mpi_send_-NOUNDERSCORE mpi_send_ MPI_SEND mpi_send

This option should normally NOT be used; configure determines what the Fortran compiler generates. This can be used to override that choice.

The option '-ar_nolocal' prevents the library archive command from attempting to use the local directory for temporary space. This option should be used when (a) there isn't much space (less than $20 \mathrm{MB}$ ) available in the partition where mpich resides and (b) there is enough space in /tmp (or wherever ar places temporary files by default).

The option '-noranlib' causes the 'ranlib' step (needed on some systems to build an object library) to be skipped. This is particularly useful on systems where 'ranlib' is optional (allowed but not needed; because it is allowed, configure chooses to use it just in case) but can fail (some 'ranlib's are implemented as scripts using 'ar'; if they don't use the local directory, they can fail (destroying the library in the process) if the temporary directory (usually '/tmp') does not have enough space. This has occurred on some OSF systems.

The option '-memdebug' enables extensive internal memory debugging code. This should be used only if you are trying to find a memory problem (it can be used to help find memory problems in user code as well).

The option '-rsh' allows you to select an alternative remote shell command (by default, configure will use 'rsh' or 'remsh' from your 'PATH'). If your remote shell command does not support the '-l' option (some AFS versions of 'rsh' have this bug), also give the option '-rshnol'. These options are useful only when building a network version of MPICH (e.g., '-device=ch_p4' or '-device=ch_tcp').

Special Tuning Options:

There are a number of options for tuning the behavoir of the ADI (Abstract Device Interface), which is the low-level message-passing interface. These should NOT be used unless you are sure you know what you are doing.

The option '-nodevdebug' disables the debugging code in the MPI ADI code. This should be used only when you are sure that everything is working correct1y. (This option is also present to remind benchmarkers that the lowlevel code by default may contain debugging code.) Note also that some of the Inewpage device code (in mpid/*) has had the debugging code removed from the source 
code. '-devdebug' turns on the debugging code. '-nodevdebug' is the default.

The option '-var_pkt' allows you to set the message size at which MPICH changes from its short to long message protocol.

The option '-pkt_size=LENGTH' allows you to choose the message length at which the ADI (Abstract Device Interface) switches from its short to long message format. LENGTH must be positive.

The option '-adi_collective' allows the ADI to provide some collective operations in addition to the basic point-to-point operations. Currently, most systems do not support this option (it is ignored) and on the others it has not been extensively tested. The option '-adi_coll_world' asks the ADI to try to use any collective operations that are supported only on the MPI_COMM_WORLD communicator (and any communicator with a similiar group). This is also untested.

Sample Configure Usage:

To make for running on Sun4's running Sunos with ch_p4 as the device, and with the installation directory equal to the current directory:

$$
\begin{aligned}
& . / \text { configure }- \text { device }=c h-p 4-\text { arch }=\text { sun } 4 \\
& \text { make }
\end{aligned}
$$

Known devices are chameleon,

ch_nx (native Intel NX calls),

ch_mpl (native IBM EUI or MPL calls),

ch_nc (native nCUBE calls, requires -arch=ncube),

ch_cmmd (native TMC CM-5 CMMD calls),

ch_p4 (p4)

ch_nexus (Nexus)

ch_meiko (for Meiko CS2, using NX compatibility library),

ch_shmem (for shared memory systems, such as SMPs),

ch_lfshmem(for shared memory systems, such as SMPs; uses lock-free message buffers),

ch_cenju3 (native NEC Cenju-3 calls),

meiko (for Meiko CS2, using elan tport library), and

$\mathrm{nx}$ (for Intel Paragon),

t3d (for the Cray T3D, using Cray shmem Iibrary).

Known architectures include (case is important)

sun4 (SUN OS $4 . x$ )

solaris (Solaris)

solaris86 (Solaris on Intel platforms)

hpux (HP UX)

rs6000 (AIX for IBM RS6000) 


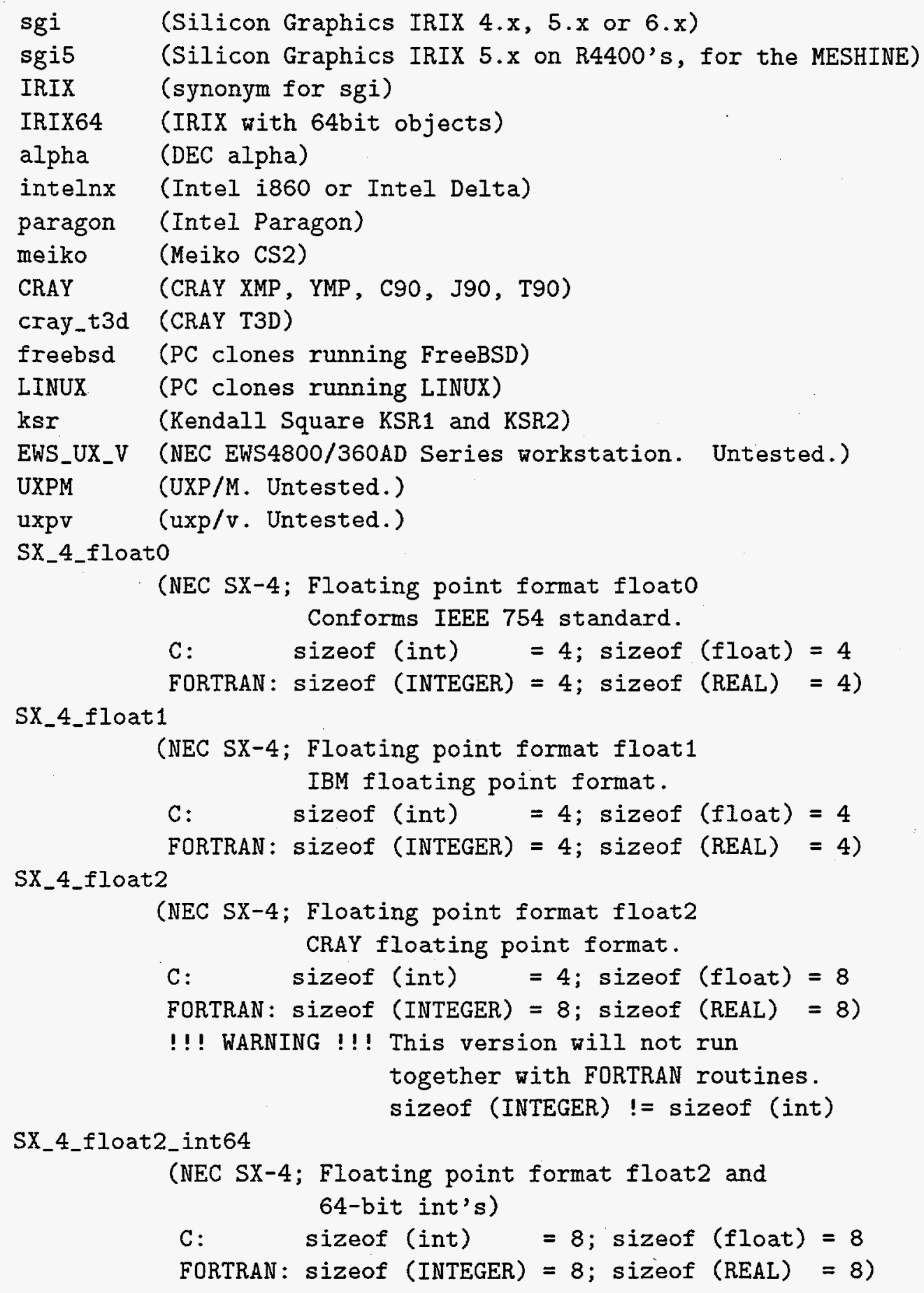

Special notes:

For SGI (-arch=IRIX) multiprocessors running the ch_p4 device, use -comm=ch_p4 to disable the use of the shared-memory $\mathrm{p} 4$ communication device, and -comm=shared to enable the shared-memory p4 communication device. The default is to enable the shared-memory communication device.

Others may be recognized. 
Normally, you should use configure with as few arguments as you can. For example, setting the $\mathrm{C}$ compiler with $-\mathrm{cc}=\mathrm{xxx}$ may require also setting -cflags=yyy; configure will (usually) choose both the compiler and flags appropriately.

mpich is implemented using an abstract device specification (ADI), described in [3]. In some environments, this abstract device is configured to be the native communication subsystem of the machine. This is done with the device argument to configure. For the rest of the environments, a generic communication device is constructed using $\mathrm{p} 4[1,2]$ and that is used as the instantiation of the ADI. In these cases, use ch_p4 as the device.

A new device named ch_nexus and based on the Nexus run-time system has been made available recently. Like $\mathrm{p} 4$, Nexus is able to use multimethod communication on many platforms. For example, the IBM SPx binary can communicate via MPL or TCP depending on which node it is communicating with. See http://www.mcs.anl.gov/people/geisler/projects/ newmpi.html for more details.

The ARCH_TYPE specifies what kind of processor the compilations will take place on. Valid ones are listed above. For the IBM SP1 and SP2, the architecture type is rs6000. If the type is not given, configure will attempt to determine it.

Some machines have multiple communication options, which are specified with the comm argument. Currently, only the ch_p4 device makes use of this. By selecting -comm=shared, a p4 device that permits the use of both shared memory and IP/TCP is built. This is particularly useful on clusters of symmetric multiprocessors.

Some sample invocations of configure are shown below. In most cases, you may wish to add the argument -mpe to the configure command; this makes the MPE extensions available to your users.

First, for massively parallel processors (MPPs) and multiprocessors:

Convex Exemplar For a Convex Exemplar, please get the official version from Convex/HP. This is based on mpich, but has been tuned for better performance on the Exemplar. If for some reason you wish to use the shared-memory version of mpich on the Convex, use

$$
\text { configure -device=ch_shmem - arch=hpux }
$$

Cray multiprocessor (not a CRAY T3D but, for example, a 4-processor Cray YMP or C90)

$$
\text { configure }- \text { device }=c_{-} \text {p }_{4}-\operatorname{arch}=\mathrm{CRAY}
$$

Cray t3d (assuming you are logged into the YMP front end).

configure - device $=t 3 d-$ arch $=c r a y \_t 3 d$

Intel Paragon configure -device=nx -arch=paragon

IBM SP2 (using the high-performance switch for communication)

configure -device=ch_mpl -arch=rs6000 
To use this, you must have POE installed.

Meiko CS-2 configure -device=ch_meiko -arch=meiko

SGI multiprocessors configure -device=ch_shmem

See the comments under SGI workstations for different 32- and 64-bit options.

For networks of workstations (can interoperate with other types of workstations),

DEC Alpha To get the full advantages of ANSI C, you may need to add -cflags="-std". For strict ANSI C, use-cflags="-std1".

IBM RS6000 In order to get the advantages of ANSI C, you may need to add -cflags="-qlanglvl=ansi". The mpich code uses _-STDC_- to check for the presence of ANSI C features; the IBM RS/6000 compilers do not define this by default.

SGI configure -device=ch_p4

Some SGI systems support both 32- and 64-bit pointers (addresses). mpich uses the architecture IRIX to refer to 32-bit systems and IRIX64 for 64-bit systems. You can use $-\operatorname{arch}=I R I X$ or $-\operatorname{arch}=I R I X 64$ to force a particular system. If you need to generate a particular version that corresponds to the $-32,-n 32$, or -64 compiler/linker options on SGI, use the architectures IRIX32, IRIXN32, or IRIX64, respectively, instead of sgi.

SGI multiprocessor (such as an Onyx, Challenge, or Power Challenge), using the shared memory for fast message passing

configure -device=ch_p4 - comm=shared

Use - arch=IRIX to force 32 -bit pointers and -arch=IRIX64 to force 64 -bit pointers.

Sun SunOS including the mpe libraries (See the users guide [5]:

configure -device=ch_p4 -arch=sun4 -mpe

Sun Solaris configure -device=ch_p4 -arch=solaris -mpe

DEC Alpha configure -device=ch_p4 -arch=alpha

FreeBSD For a network of PC's running the FreeBSD version of Unix:

configure - device $=c h-p 4-\operatorname{arch}=$ freebsd

Nexus device For a machine using the Nexus device, change the device in the above examples to ch_nexus. The Nexus device requires an ANSI C compiler, because the Nexus header files use the function prototypes. If the default compiler does not support function prototypes (assuming ansicc is your compiler), add -cc=ansicc to the configure command line. Remember to point this at the correct compiler for your message-passing system (e.g., mpcc for the SP or icc for the Paragon). 
HP HPUX For a network of HP's, including the mpe library but leaving out of it the MPE $\mathrm{X}$ graphics routines:

$$
\text { configure -device=ch_p4 -arch=hpux -mpe -no_mpegraphics }
$$

Fujitsu For a network of Fujitsu M780s rünning UXP/M, the following options have been tested:

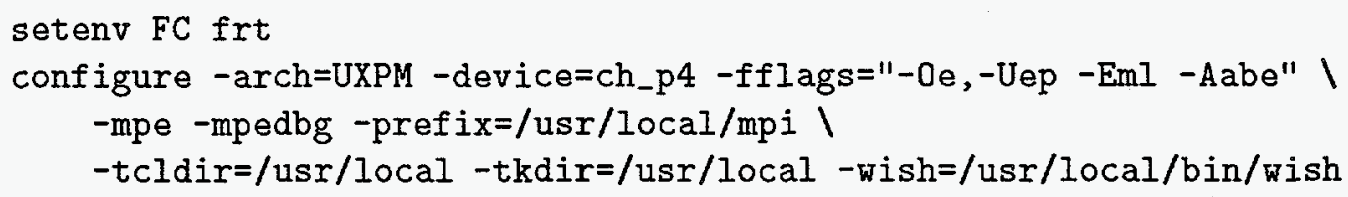

mpich can be run on a heterogeneous network of workstations of various kinds. For simple collections of workstations, the mpirun command can be used; more complex collections of heterogeneous machines require a p4 "procgroup file" (for the ch_p4 device) or a "startup file" (for the ch_nexus device). The format of these files is described in Section 6.1.

\subsection{Building a Production mpich}

By default, configure sets up mpich to be compiled without optimization and with additional code to help in identifying problems and behavior of the mpich implementation. Once mpich passes the tests (see Section 7), you may wish to rebuild mpich without the debugging code. This will produce significantly smaller libraries and slightly faster code. To do this, add the options

-opt $=-0$-nodevdebug

to the configure line, and rerun make. You may also include multiple optimization options by enclosing them in quotes:

-opt="-0 -qarch=pwr2"

\subsection{What If There Is No Fortran Compiler?}

The configure program should discover that there is no Fortran compiler. You can force configure to not build the Fortran parts of the code with the option -nof 77. In this case, only the $\mathrm{C}$ programs will be built and tested.

\subsection{Special Issues for Heterogeneous Networks}

When building mpich for a heterogeneous collection of workstations, you may wish to configure with the option -no_short_longs. This indicates to mpich that it should not provide support for the $\mathrm{C}$ type long double. This can improve performance between systems that have the same datatype lengths for all other types (some Intel x86 machines have 12-byte long doubles; many other systems use either 8- or 16-byte long doubles). 


\section{Compiling mpich}

Once configure has determined the features of your system, all we have to do now is

make

This will clean all the directories of previous object files (if any), compile both profiling and nonprofiling versions of the source code, build all necessary libraries, and link both a sample Fortran program and a sample $\mathrm{C}$ program as a test that everything is working. If anything goes wrong, check Section 13 to see whether anything is said there about your problem. If not, follow the directions in Section 13.1 for submitting a bug report. To simplify checking for problems, use

make >\& make. $\log \&$

Specific (nondefault) targets can also be made. See the Makefile to see what they are.

After this make is run, the size of the distribution will be about 20 megabytes (depending on the particular machine it is being compiled for), before any of the examples or the extensive test library is built. The Makefiles are built for the various example subdirectories, but the example programs have to be made by hand.

\subsection{Getting tcl, tk, and wish}

The software packages $t c l, t k$, and wish are available by anonymous ftp from $f t p . s m l i . c o m$ in the directory /pub/tcl. They are needed only for the upshot and nupshot programs; you do not need them in order to install MPI.

You should get tcl7.3.tar.Z and tk3.6.tar.Z (and patch tk3.6p1.patch). Later versions of both $\mathrm{tcl}$ and tk are incompatible with these and do not work with nupshot. The upshot program has been modified to work with either tk 3.6 or tk4.0.

It is necessary that the wish program be accessible to users; the other parts of tcl and tk do not need to be installed (but make sure that everything that wish itself needs is installed).

\subsection{Building Multiple Devices or Architectures}

When building more than one version of mpich, it is important to use the versions of the programs mpirun, etc., from the library directory, not mpich/bin.

\section{Running an MPI Program}

In order to make running programs on parallel machines nearly as portable as writing them, the environment distributed with mpich contains a script for doing so. It is the 
mpirun command, found in the mpich/bin directory. Several of the examples directories already have symbolic links to this command, but eventually you might wish to add it to your path, with (assuming your shell is the $\mathrm{C}$ shell)

set path $=(\$$ path $/$ home $/ \mathrm{me} / \mathrm{mpich} / \mathrm{bin})$

More details on mpirun can be found in Section 11.2. If you are going to run on a network of workstations, you will need a machines.xxxx file in mpich/util/machines; see Section 6.1 for details. Systems that use various kinds of filesystem automounters may need to make small changes to these programs; these are detailed in Section 6.1.1.

Some simple MPI programs will have been built during the compilation process. They are in the directory mpich/examples/basic and contain a $\mathrm{C}$ and a Fortran program for estimating $\pi$. Change to that directory, and do

mpirun -np $4 \mathrm{cpi}$

to run the $\mathrm{C}$ version, and

mpirun -np 4 fpi

to run the Fortran version. At this point, you have minimally tested your installation of mpich. You might also wish to check the performance of MPI on your system. You can do a crude check by running the program systest, also found in the examples/basic directory. To try it, do

make systest

mpirun -np 2 systest

For a more precise benchmark, see Section 10.

Another program in the examples/basic subdirectory is cpilog. This program uses some of the routines from the MPE library; you must have configured with the -mpe option. If you make it and run it, it will produce a simple log file that can be viewed with the program analysis tool upshot. To do so, you may need to build upshot with

make upshot

in the top level mpich directory. Note that upshot requires the th shell wish. To use upshot to view a $\log$ file, do

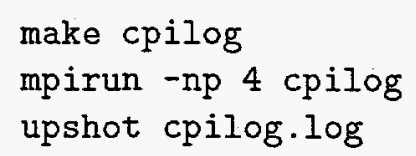

The log file produced by cpilog is not very interesting, since cpi is such a simple program. Many interesting logfiles can be found in the profiling/upshot/logfiles subdirectory. 
The file cpilog.c demonstrates how to instrument your own code for producing such logs. The users guide [5] describes how to link with a version of mpich that produces them automatically. For a short description of the programs in the examples/basic directory, see the README file there. The logging routines are part of mpe, so be sure that your configuration has been done with the -mpe option.

\subsection{Special Considerations for Running on a Network of Workstations}

To run on a network of workstations, you must specify in some way the host names of the machines that you wish to run on. This process can be done in several ways. These are described in detail in the users guide. We give a shorter version here.

The easiest way is to edit the file mpich/util/machines/machines.xxxx, to contain names of machines of architecture $x x x x$. The $x x x x$ matches the arch given when mpich was configured. Then whenever mpirun is executed, the required number of hosts will be selcted from this file for the run. (There is no fancy scheduling; the hosts are selected starting from the top.) To run all your MPI processes on a single workstation, just make all the lines in the file the same. A sample machines.sun4 file might look like

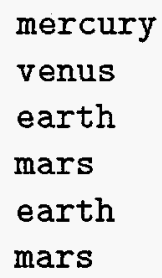

To run the test suite in examples/test, you need a machines file with at least five lines in it. This is for homogeneous networks. Heterogeneous networks are discussed in the users guide.

\subsubsection{Dealing with Automounters}

Automounters are programs that dynamically make file systems available when needed. While very convenient, many automounters are unable to recognize the file system names that the automounter itself generates. For example, if a user accesses a file /home/me, the automounter may discover that it needs to mount this file system, and does so in /tmp_mnt/home/me. Unfortunately, if the automounter on a different system is presented with /tmp_mnt/home/me instead of /home/me, it may not be able to find the file system. This would not be such a problem if commands like pwd returned /home/me instead of /tmp_mnt/home/me; unfortunately, it is all too easy to get a path that the automounter should, but does not, recognize.

To deal with this problem, configure allows you to specify a filter program when you configure with the option -automountfix=PROGRAM, where PROGRAM is a filter that reads a file path from standard input, makes any changes necessary, and writes the output to standard output. By default, the value of PROGRAM is

sed -e s@/tmp_mnt/@/@g 
This uses the sed command to strip the string /tmp_mnt from the file name. Simple sed scripts like this may be used as long as they do not involve quotes (single or double) or use $\%$ (these will interfere with the shell commands in configure that do the replacements). If you need more complex processing, use a separate shell script or program.

As another example, some systems will generate paths like

/a/thishost/root/home/username/....

which are valid only on the machine thishost, but also have paths of the form

/u/home/username/....

that are valid everywhere. For this case, the configure option

-automountfix='sed -e s@/a/.1*/home@/u/home@g'

will make sure that mpirun gets the proper filename.

\subsubsection{Faster Job Startup}

When using the ch_p4 or ch_nexus devices, it is possible to speed the process of starting jobs by using the secure server. The secure server is a program that runs on the machines listed in the machines.ARCH file and that allows programs to start faster. There are two ways to install this program: so that only one user may use it and so all users may use it. No special privileges are required to install the secure server for a single user's use.

To use the secure server, follow these steps:

1. Choose a port. This is a number that you will use to identify the secure server (different port numbers may be used to allow multiple secure servers to operate). A good choice is a number over 1000 . If you pick a number that is already being used, the server will exit, and you will have to pick another number. On many systems, you can use the rpcinfo command to find out which ports are in use (or reserved). For example, to find the ports in use on host mysun, try

rpcinfo -p mysun

2. If using the ch_p4 device, build the secure server. From the top-level directory, do make serv_p4

At the end of this step, the executable for the secure server is in the same directory as the MPI libraries.

3. Start the secure server. The script bin/chp4_servs

$$
\mathrm{bin} / \mathrm{chp} 4 \text { _servs }- \text { port }=\mathrm{n}-\operatorname{arch}=\$ \mathrm{ARCH}
$$


can be used to start the secure servers. This makes use of the remote shell command (rsh or remsh) to start the servers; if you cannot use the remote shell command, you will need to $\log$ into each system on which you want to start the secure server and start the server manually. The command to start an individual server using port 2345 is

serv_p4 -o $-p 2345$ \&

For example, if you had chosen a port number of 2345 and were using sun 4 s, you would give the command

$$
\text { bin/chp4_servs }- \text { port }=2345-\operatorname{arch}=\operatorname{sun} 4
$$

The server will keep a $\log$ of its activities in a file with the name P4Server.Log. $x x x x$ in the current directory, where $\mathrm{xxxx}$ is the process id of the process that started the server (note that the server may be running as a child of that initial process).

4. To make use of the secure servers using the ch_p4 device, you must inform mpirun of the port number. You can do this in two ways. The first is to give the -p4ssport $n$ option to mpirun. For example, if the port is 2345 and you wish to run $\mathrm{cpi}$ on four processors, use

$$
\text { mpirun -np } 4 \text {-p4ssport } 2345 \mathrm{cpi}
$$

The other way to inform mpirun of the secure server is to use the environment variables MPI_USEP4SSPORT and MPI_P4SSPORT. In the C-shell, you can set these with

setenv MPI_USEP4SSPORT yes

setenv MPI_P4SSPORT 2345

The value of MPI_P4SSPORT must be the port with which you started the secure servers. When these environment variables are set, no extra options are needed with mpirun.

5. If using the ch_nexus device, find the Nexus secure server in the Nexus directory, for example, /usr/local/nexus/bin/sserver.

6. Start the Nexus secure server on each machine. The command to start an individual server using port 2345 is

ssserver $-d-p 2345 \&$

7. The ch_rexus device requires that you record the port numbers in a resource database $(. \mathrm{rdb})$ file. The format of the file is

$$
\text { 〈host> ss_port=<port \#> }
$$

The -nexusdb flag should be used to tell mpirun the name of the file:

$$
\text { mpirun -nexusdb ports program }
$$

Note that when mpich is installed, the secure server and the startup commands are copied into the library directory so that users may start their own copies of the server. This is discussed in the users guide. 


\subsubsection{Stopping the Servers}

To stop the servers, their processes must be killed. Stopping is easily done with the Scalable Unix Tools [4] with the command

pfps -all -tn serv_p4 -and -o \$LOGNAME -kill INT

Alternately, you can $\log$ into each system and execute something like

ps auxww I egrep '\$LOGNAME。*serv_p4'

and then use the kill command on the resulting process number (users of System V-style ps commands will have to figure out what their particular form of ps needs and adjust the egrep command accordingly).

An alternative approach is discussed in Section 6.1.4.

\subsubsection{Managing the Servers}

An experimental per 15 program is provided to help you manage the p4 secure servers. This program is chkserv, and is in the util directory. You can use this program to check that your servers are running, start up new servers, or stop servers that are running.

Before using this script, you must edit it. It has sample values for the fields that it will use. In particular, you should set serv_p4, portnum, and machinelist appropriately; you may also need to set the first line to your version of perl5.

To check on the status of your servers, use

chkserv -port 2345

To restart any servers that have stopped, use

chkserv -port 2345 -restart

This does not restart servers that are already running; you can use this as a cron job every morning to make sure that your servers are running. Note that this uses rsh to start the process on the remote systems; if you can't use rsh, you'll need to restart the servers by hand. In that case, you can use the output from chkserv -port 1234 to see which servers need to be restarted.

chkserv -port $2345-k i l l$

This contacts all running servers and tells them to exit. It does not use rsh, and can be used on any system (it contacts the server and tells it to exit).

This software is experimental. If you have comments or suggestions, please send them to mpibugs@mcs . anl.gov. 


\subsection{Special Considerations for Running with Shared Memory}

When using the ch_shmem or ch_If shmem devices with System V shared memory, processes that exit abnormally (e.g., with a segmentation violation) may leave System $V$ semaphores or shared-memory segments allocated. ${ }^{2}$ Since there are usually a limited number of these objects, it is important to recover them. The Unix command ipcs can be used to list the allocated semaphores and shared memory segments, and ipcrm can be used to delete them. The script bin/cleanipcs can be used to identify and delete all System V IPCs owned by the calling user; the use is simply

$$
\text { bin/cleanipcs }
$$

\section{Thorough Testing}

The examples/test directory contains subdirectories of small programs that systematically test a large subset of the MPI functions. The command

$$
\text { make testing }
$$

in the mpich/examples/test directory will cause these programs to be compiled, linked, and executed, and their output to be compared with the expected output. Linking all these test programs takes up considerable space, so you might wish to do

make clean

in the test directory afterwards. The individual parts of MPI (point-to-point, collective, topology, etc.) can be tested separately by

$$
\text { make testing }
$$

in the separate subdirectories for examples/test.

If your disk space is limited, consider either running with

$$
\text { make testing TESTARGS }=-\operatorname{small}
$$

or going to each directory and executing

$$
\text { /runtests -small }
$$

With the -small switch, each executable is built, run, and deleted before the next test program is built.

If you have a problem, first check the troubleshooting guides and the lists of known problems. If you still need help, send detailed information to mpi-bugs@mcs . anl.gov.

\footnotetext{
${ }^{2}$ The System V IPC (interprocess communication) mechanisms do not have a "delete on unreferenced" attribute.
} 


\section{Installing mpich for Others to Use}

This step is optional. Once you have tested all parts of the MPI distribution (including the tools, particularly upshot and/or nupshot), you can install mpich into a publicly available directory. To install the libraries and include files in a publicly available place, choose a directory, such as /usr/local/mpi, change to the top-level mpich directory, and do

make install PREFIX=/usr/local/mpi

The man pages will have been copied with the installation, so you might wish to add /usr/local/mpi/man to the default system MANPATH. The man pages can be conveniently browsed with the mpiman command, found in the mpich/bin directory.

A good way to handle multiple releases of mpich is to install them into directories whose names include the version number and then set a link from mpi to that directory. For example, if the current version is 1.0 .13 , the installation commands to install into /usr/local are

make install PREFIX=/usr/local/mpi-1.0.13

$\mathrm{rm} / \mathrm{usr} /$ local/mpi

In -s /usr/local/mpi-1.0.13/usr/local/mpi

The script util/mpiinstall provides more control over the installation of mpich (in fact, make install just runs this script). For example, you can change the protection of the files when they are installed with the options -mode=nnnn (for regular files) and -xmode=nnnn (for executables and directories). You can set the directory into which the man pages will be placed with -manpath $=\langle$ path $\rangle$. The option -help shows the full set of options for mpiinstall.

Installing nupshot can sometimes be troublesome. You can use the switch -nonupshot to mpiinstall to not install nupshot; alternately, you can use the switch -cpnuphost to install the copy in mpich/profiling/nupshot. Normally, mpiinstall builds a new version of nupshot to ensure that all of the paths are correct (nupshot needs to find files where it is installed). If you need to manually build nupshot for installation, the -cpnupshot switch will allow you to install that version.

If you are supporting multiple devices on a single platform (for example, ch_p4 and ch_shmem), you should build one and install it in the regular way, then build the second and install it with mpiinstall -libonly. In this case, make sure that your users use the programs in the library directory rather than the bin directory.

\subsection{User Commands}

The commands mpirun, mpicc, mpif77, mpiman, and mpireconfig should be in the user's search path. Note that if several architectures and/or mpich devices are supported, it is important that the correct directory be added to the user's path. For convenience, these are in both mpich/bin and mpich/lib/ $\langle$ arch $\rangle /\langle$ device $\rangle$; if there is any chance that multiple architectures or devices are used, the second form should be used. 


\subsection{Installing Documentation}

The mpich implementation comes with several kinds of documentation. Installers are encouraged to provide site-specific information, such as the location of the installation (particularly if it is not in /usr/local/mpi).

\subsubsection{Man Pages}

A complete set of Unix man pages for the mpich implementation are in mpich/man. $\operatorname{man} / \operatorname{man} 3$ contains the MPI routines; $\operatorname{man} / \operatorname{man} 4$ contains the MPE routines and mpirun, and man/man5 contains the MPID routines (these are for the low-level part of the mpich implementation, are are not of interest to users). The command mpich/bin/mpiman is a script that runs xman on these man pages.

\subsubsection{Web Versions of Man Pages}

Web (HTML) versions are available from ftp://info.mcs.anl.gov/pub/mpi/manwww.tar.Z.

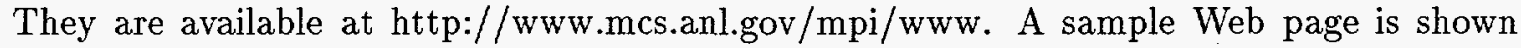
below and is also available in mpich/util/mpichsite.html

<TITLE>Using MPICH at Argonne</TITLE>

$<$ H1 Site-specific information on the MPICH implementation of MPI</H1>

$\langle\mathrm{H} 2\rangle$ Location of libraries $</ \mathrm{H} 2\rangle$

The MPICH implementation is located in $\langle\mathrm{TT}\rangle / \mathrm{usr} / \mathrm{local} / \mathrm{mpi} / \mathrm{lib}\langle\mathrm{TT}\rangle$; the libraries are in

$\langle\mathrm{DL}\rangle$

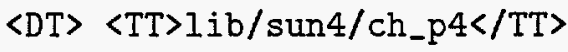

<DD> for Sun4

$\langle\mathrm{DT}\rangle\langle\mathrm{TT}\rangle \mathrm{lib} / \mathrm{rs} 6000 / \mathrm{Ch}_{-} \mathrm{mpl}\langle/ \mathrm{TT}\rangle$

$\langle D D\rangle$ for the SPX

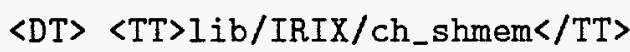

$\langle D D\rangle$ for the SGI system

$\langle/ D L\rangle$

$<$ H2>Location of programs</H2>

Programs are located in the same directory as the libraries

$<$ H2>Documentation</H2>

The command $\langle\mathrm{TT}\rangle / \mathrm{usr} /$ local/mpi/bin/mpiman</TT> provides man pages with xman.

The <A HREF="http://ww.mcs.anl.gov/mpi/www/index.html">man pages $\langle/ A\rangle$

are also available. The

<A HREF="http://www.mcs.anl.gov/mpi/mpi-report-1.1/mpi-report.html">MPI

Standard Version $1.1\langle/ \mathrm{A}\rangle$ is available in hypertext form. 
$\langle$ H2 $>$ Examples $\langle/ \mathrm{H} 2\rangle$

A simple example in $\mathrm{C}$ and Fortran is in $\langle\mathrm{TT}\rangle / \mathrm{usr} / \mathrm{local} / \mathrm{mpi} / \mathrm{examples}\langle\mathrm{TT}\rangle$. More examples may be found in the MPICH source tree, located at $\langle\mathrm{TT}\rangle /$ home/MPI/mpich/examples</TT $\rangle$.

\subsubsection{Examples}

Users often prefer working from example Makefiles and programs. The directory that is installed in the examples directory contains a $\mathrm{C}$ and Fortran version of the 'pi' program, along with a Makefile.in. Users may be interested in some of the examples that are in the source tree, also in the examples directory.

\section{Internationalization}

mpich has support for providing error messages in different languages. This makes use of the X/Open message catalog services, which are a standard way of providing multilanguage support. This multilanguage support is often called NLS, for National Language Support. mpich comes with error messages in English; additional languages will be provided as we get the translations (if you wish to provide one, please send mail to mpi-bugs@mes.anl.gov). More precisely, mpich uses an English version that uses the ISO Latin-1 character set (ISO8859-1). We expect to provide other versions that also use the Latin-1 character set, subject to getting translations of the messages.

To create a new message catalog, copy the file mpich.En_US.msg to a file mpich.mylanguage.msg and translate the entries. The value of mylanguage should match the ones used for your system, for example, mpich.De_DE.msg for German. Many systems put their NLS files in /usr/lib/nls/msg; you can also check the value of the environment variable NLSPATH on your system. Note that some systems provide the routines and programs to support NLS, but do not make use of it and do not provide an initial NLSPATH value.

For emacs users, check the emacs info under "European Display". The commands

M-x standard-display-european

$\mathrm{M}-\mathrm{x}$ iso-accents-mode

can be used to input most European languages. You can also load iso-transI and use C-x 8 to compose characters (this sets the high bit in the character). mpich currently does not support languages that require multibyte character sets (such as Japanese). However, the only changes needed are in the file src/env/errmsg.c; if you are interested in developing a multibyte character set version, please let us know.

By default, mpich uses the value of NLSPATH to find the message catalogs. If this fails, it tries MPICHNLSPATH, and if that fails, it uses English language versions that are coded into the library. 
The catalogs are not, however, installed into these directories. Instead, you will find them in the library directory for a particular architecture; for example, mpich/lib/rs6000.

\section{Benchmarking mpich}

The mpich/examples/perftest directory contains a sophisticated tool for measuring latency and bandwidth for mpich on your system. To run it, first make sure that mpich was configured with the -mpe option. Then go to mpich/examples/perftest and do

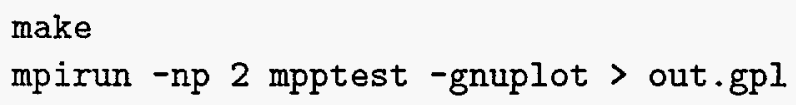

The file out.gpl will then contain the necessary gnuplot commands. The file mppout.gpl will contain the data. To view the data with gnuplot, use

gnuplot out.gpl

or use

load 'out.gpl'

from within gnuplot. Depending on your environment and version of gnuplot, you may need to start gnuplot first and issue the command set terminal $\mathrm{x} 11$ before executing load 'out.gpl'.

The programs mpptest and goptest have a wide variety of capabilities; the option -help will list them. For example, mpptest can automatically pick message lengths to discover any sudden changes in behavior and can investigate the ability to overlap communication with computation. These programs are written using MPI and may be used with any MPI implementation, not just mpich.

\section{The mpich Programming Environment}

\subsection{Introduction}

The MPI standard specifies nothing outside of MPI programs, not even how they will be started. mpich supplies a number useful tools for managing MPI programs, including

1. mpirun, a portable startup command, so that MPI programs can be started the same way in many different environments,

2. mpicc and mpif77, easy ways to compile and link MPI programs.

3. mpireconfig, a way to create Makefiles from Makefile.in templates 
4. mpe, a library of useful routines that work will with MPI. Curently this library includes both routines for producing log files of time-stamped events and a simple parallel $\mathrm{X}$ graphics library, routines for providing a sequential section code, and routines to start a debugger when errors occur.

5. A set of predefined profiling libraries. The MPI standard specifies a mechanism whereby the user may "wrap" any collection of MPI functions with code of his own, without accessing the MPI implementation source code. We supply tools for constructing such a profiling version of the MPI library with a minimum of effort, as well as three preconstructed sets of wrappers, for accumulating time spent in MPI routines, for preparing log files, and for program animation.

6. upshot, a tool for examining log files produced by the mpe logging functions or by the automatic logging in the logging profiling library.

7. nupshot, an experimental, faster version of upshot.

\section{2 mpirun, a Portable Startup Script}

Each parallel computing environment provides some mechanism for starting parallel programs. Unfortunately, these mechanisms are very different from one another. In an effort to make this aspect of parallel programming portable as well, mpich contains a script called mpirun. This script is partially customized during the configuration process when mpich is built. Therefore the actual "source" for mpirun is in the file mpirun.sh. in in the mpich/bin directory. The most common invocation of mpirun just specifies the number of processes and the program to run:

$$
\text { mpirun -np } 4 \mathrm{cpi}
$$

The complete list of options for mpirun is obtained by running

$$
\text { mpirun -help }
$$

This is the result:

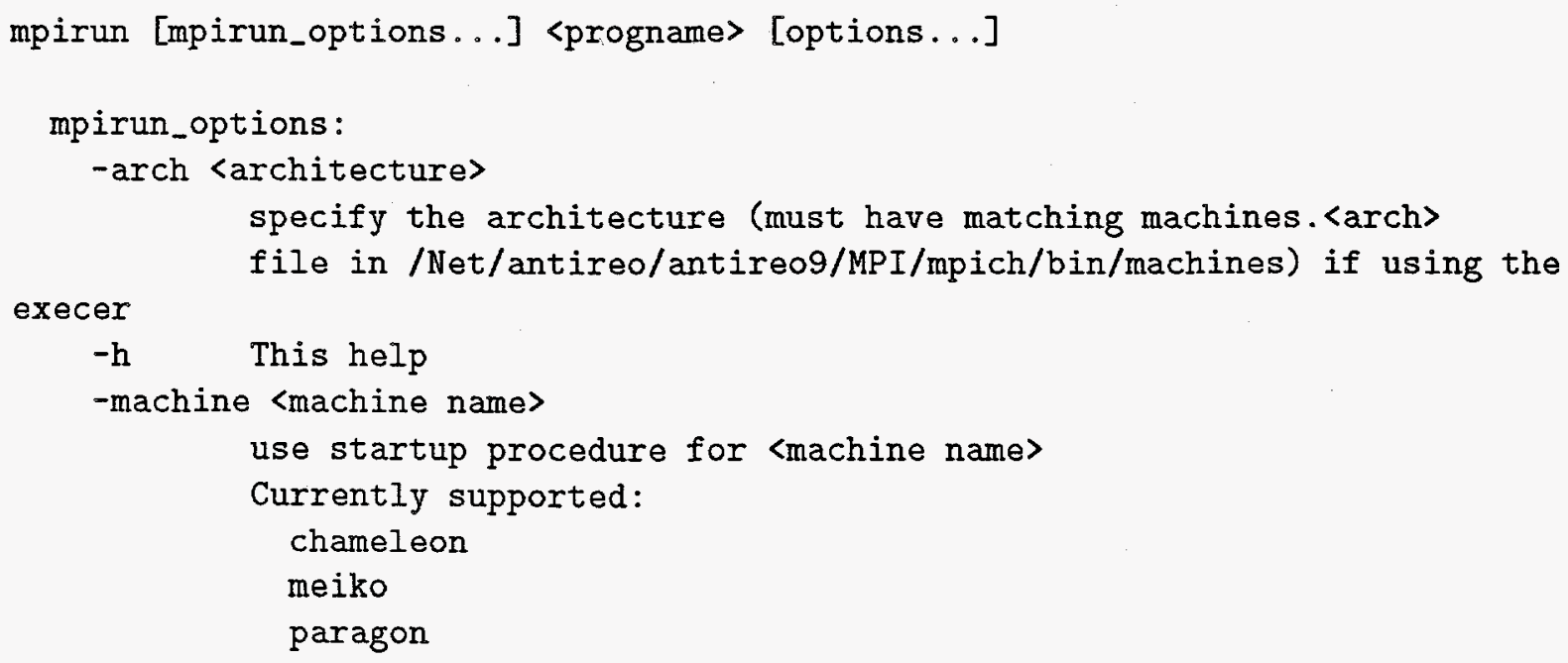




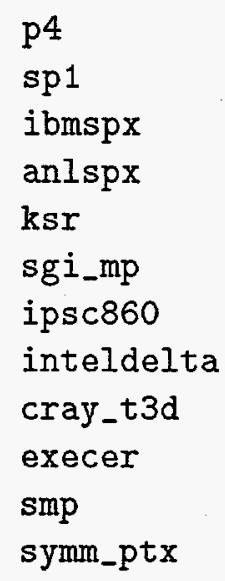

Special Options for NEC - CENJU-3:

-batch Excecute program as a batch job (using cjbr)

-stdout filename

Use filename as the standard output for the program.

-stderr filename

Use filename as the standard error for the program.

Special Options for Nexus device: 
-nexuspg filename

Use the given Nexus startup file instead of creating one.

Dverrides -np and -nolocal, selects -leave_pg.

-nexusdb filename

Use the given Nexus resource database.

Special Options for Workstation Clusters:

-e Use execer to start the program on workstation clusters

-pg Use a procgroup file to start the p4 programs, not execer (default)

-leave_pg

Don't delete the P4 procgroup file after running

-p4pg filename

Use the given p4 procgroup file instead of creating one.

Dverrides -np and -nolocal, selects -leave_pg.

-tcppg filename

Use the given tcp procgroup file instead of creating one.

Overrides -np and -nolocal, selects -leave_pg.

-p4ssport num

Use the p4 secure server with port number num to start the

programs. If num is 0 , use the value of the

environment variable MPI_P4SSPORT. Using the server can speed up process startup. If MPI_USEP4SSPORT as well as MPI_P4SSPORT are set, then that has the effect of giving mpirun the -p4ssport 0 parameters.

Special Options for Batch Environments:

-mvhome Move the executable to the home directory. This

is needed when all file systems are not cross-mounted

Currently only used by anlspx

-mvback files

Move the indicated files back to the current directory.

Needed only when using -mvhome; has no effect otherwise.

-maxtime min

Maximum job run time in minutes. Currently used only

by anlspx. Default value is 15 minutes.

-nopoll Do not use a polling-mode communication.

Available only on IBM SPx.

-mem value

This is the per node memory request (in Mbytes). Needed for some

CM-5s. ( Default 32.)

-cpu time 
This is the the hard cpu limit used for some CM-5s in minutes. (Default minutes.)

Special Options for IBM SP2:

- cac name

CAC for ANL scheduler. Currently used only by anlspx.

If not provided will choose some valid $\mathrm{CAC}$.

Special Options for Intel Paragon:

-paragontype name

Selects one of default, mkpart, NQS, depending on how you want to submit jobs to a Paragon.

-paragonname name

Remote shells to name to run the job (using the -sz method) on a Paragon.

-paragonpn name

Name of partition to run on in a Paragon (using the -pn name command-line argument)

On exit, mpirun returns a status of zero unless mpirun detected a problem, in which case it returns a non-zero status (currently, all are one, but this may change in the future).

Multiple architectures may be handled by giving multiple -arch and -np arguments. For example, to run a program on 2 sun $4 \mathrm{~s}$ and $3 \mathrm{rs} 6000 \mathrm{~s}$, with the local machine being a sun 4 , use

/home/MPI/mpich/util/mpirun -arch sun4 -np 2 -arch rs6000 -np 3 program

This assumes that program will run on both architectures. If different executables are needed, the string ' $\% \mathrm{a}$ ' will be replaced with the arch name. For example, if the programs are program.sun 4 and program.rs6000, then the command is

/home/MPI/mpich/util/mpirun -arch sun4 -np 2 -arch rs6000 -np 3 program.\%a

If instead the executables are in different directories; for example, /tmp/me/sun 4 and /tmp/me/rs6000, then the command is

/home/MPI/mpich/util/mpirun -arch sun4 -np 2 -arch $\mathrm{rs} 6000$-np $3 / \mathrm{tmp} / \mathrm{me} / \%$ a/program

It is important to specify the architecture with -arch BEFORE specifying the number of processors. Also, the FIRST -arch command must refer to the processor on which the job will be started. Specifically, if -nolocal is 
NOT specified, then the first -arch must refer to the processor from which mpirun is running.

For backward compatibility with earlier versions of mpirun, each of these arguments can also be used with the prefix $\mathrm{mr}_{-}$, as in

$$
\text { mpirun -mr_np } 4 \text { myprog }
$$

\subsection{The mpicc and mpif77 Commands}

The mpich implementation provides two commands for compiling and linking $\mathrm{C}$ and Fortran programs. You may use these commands instead of the Makefile.in versions, particularly for programs contained in a small number of files. In addition, they have a simple interface to the profiling and visualization libraries described in [7]. This is a program to compile or link MPI programs. In addition, the following special options are supported:

-mpilog Build the version that generates MPE log files.

-mpitrace Build the version that generates traces.

-mpianim Build the version that generates real-time animation.

-show Show the commands that would be used without actually running them.

Use this just like the usual $\mathrm{C}$ or Fortran compiler, for example,

$$
\begin{aligned}
& \text { mpicc -c foo.c } \\
& \text { mpif77 -c foo.f }
\end{aligned}
$$

and

mpice -o foo foo.o

mpif77 -o foo foo.o

Commands for the linker may include additional libraries. For example, to use some routines from the MPE library, enter

$$
\text { mpicc to foo foo.o - Impe }
$$

Combining compilation and linking in a single command, as shown here,

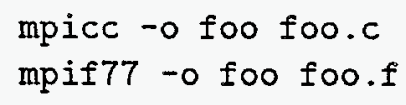

may not work on some systems, and is not recommended.

These commands are set up for a specific architecture and mpich device and are located in the directory that contains the MPI libraries. For example, if the architecture is sun 4 and the device is ch_p4, these commands may be found in /usr/local/mpi/lib/sun4/ch_p4 (assuming that mpich is installed in /usr/local/mpi). 


\section{4 mpireconfig, a Way to Create Makefiles}

Much of mpich's portability is handled throught the careful construction of system-dependent Makefiles by the configure program. This is fine for installing mpich, but what can you do when you are building a new application? For simple applications, the mpicc and mpif 77 commands may be the simplest way to build a new application. For more complex codes, we recommend taking a sample Makefile.in file, for example, in mpich/examples/test/pt2pt. Modify those parts that are are relevant, such as the EXECS and specific program targets. To create a Makefile, just execute

mpireconfig Makefile

(mpireconfig is in the same directory as mpirun). This generates a new Makefile from Makefile.in, with the correct parameters for the mpich that was installed.

\section{5 nupshot, a Way to View Logfiles}

Nupshot is a newer version of upshot [6] and can display log files created by using either the -mpilog option to mpicc or mpif77 or through use of the MPE logfile facilities.

\section{Automatic Report Generation}

We are working on a prototype system for automatically producing a report describing the installation of MPI on the system you are using. This is done by going to the top-level directory and doing

configure ...> > config.log

doc/port

Warning: this rebuilds the system, since one of the things the report will contain is the set of machine-specific parameters used and the total time it take to build it. The reason for directing the configure output to a file is so that the document generator can include comments on the configuration itself, such as calling out any problems that configure noticed.

If the system that you are running on contains all the necessary components for producing the report, it will appear in the file doc/doc1.tex. This is a LaTeX file and should be processed in the doc directory:

cd doc

latex doc1

bibtex

latex doc 1

dvips doc 1

The graphs of performance require gnuplot and are generated as Postscript files. 


\section{Problems}

This section describes some commonly encountered problems and their solutions. It also describes machine-dependent considerations. You should also check the Users Guide, where problems related to compiling, linking, and running MPI programs (as opposed to building the mpich implementation) are discussed.

\subsection{Submitting Bug Reports}

Any problem that you can't solve by checking this section should be sent to mpi-bugs@mcs anl.gov. Please include

- The version of MPICH (e.g., 1.0.13)

- The output of running your program with the -mpiversion argument (e.g., mpirun -np 1 a.out -mpiversion)

- The output of

uname -a

for your system. If you are on an SGI system, also

hinv

If the problem is with a script like configure or mpirun, run the script with the -echo argument (e.g., mpirun -echo -np 4 a.out)

If you are using a network of workstations, also send the output of bin/tstmachines. The program tstmachines is discussed in the users guide.

If you have more than one problem, please send them in separate messages; this simplifies our handling of problem reports.

The rest of this section contains some information on trouble-shooting mpich. Some of these problems are peculiar to specific environments and give suggested work-arounds. Each section is organized in question-and-answer format, with questions that relate to more than one environment (workstation, operating system, etc.) first, followed by questions that are specific to a particular environment. Problems with workstation clusters are collected together as well.

\subsection{Problems Configuring}

\subsubsection{General}

1. Q: When trying to run configure, I get error messages like

$$
\text { ./configure: syntax error at line 20: '(' unexpected }
$$


A: You have an obsolete version of the Bourne shell (sh). mpich requires that the sh shell support shell procedures; this has been standard in most Bourne shells for years. To fix this, you might consider (a) getting an update from your vendor or (b) installing one of the many publicly available sh-shell replacements.

2. Q: The configure reports the compiler as being broken, but there is no problem with the compiler (it runs the test that supposedly failed without trouble).

A: You may be using the Bash shell (/bin/bash) as a replacement for the Bourne shell $(/ \mathrm{bin} / \mathrm{sh})$. We have reports that, at least under LINUX, Bash does not properly handle return codes in expressions. One fix is to use a different shell, such as /bin/ash, on those systems.

This won't work on some LINUX systems (every shell is broken). We have reports that the following will work:

(a) In configure, change trap 'rm - $f$ confdefs*' 0 to trap 'rm -f confdefs*' 1

(b) After configure finishes, remove the file confdefs.h manually.

3. Q: configure reports errors of the form

checking gnumake... 1: Bad file number

A: Some versions of the Bash shell do not handle output redirection correctly. Either upgrade your version of Bash or run configure under another shell (such as /bin/sh). Make sure that the version of sh that you use is not an alias for Bash. configure tries to detect this situation and will normally issue an error message.

\subsubsection{LINUX}

1. Q: The configure step issues the message

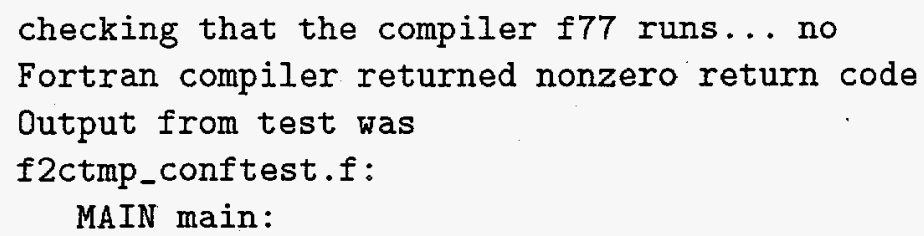

A: This is probably caused by a problem in the Fortran compiler in LINUX. The $f 77$ command in LINUX is often a shell script that uses the f2c program to convert the Fortran program to $\mathrm{C}$ and then compile it with the $\mathrm{C}$ compiler. In many versions of LINUX, this script has an error that causes a nonzero return code even when the compilation is successful.

To fix this problem, you need a corrected $f 77$ script. If you can edit the script yourself, change the last 3 lines from

case \$cOPT in 2) \$CC \$G -o \$OUTF \$OFILES - If $2 \mathrm{c}-1 \mathrm{~m}$; ; esac

$r c=\$ ?$

exit $\$ r c$ 
to

case \$COPT in 2) \$CC \$G -o \$OUTF \$OFILES - If $2 \mathrm{c}-\mathrm{lm}$; ; esac

$\mathrm{rc}=\$$ ?

trap 0

exit \$rc

2. Q: The link test fails on LINUX with messages like

overtake.o(.text+0x59): undefined reference to 'MPI_COMM_WORLD'

overtake.o(.text+0x81): undefined reference to 'MPI_COMM_WORLD'

...

A: This is probably caused by a problem in the Fortran compiler in LINUX. The $\mathbf{f 7 7}$ command in LINUX is often a shell script that uses the $\mathrm{f} 2 \mathrm{c}$ program to convert the Fortran program to $\mathrm{C}$ and then compile it with the $\mathrm{C}$ compiler. In many versions of LINUX, this script has an error that causes a nonzero return code even when the compilation is successful.

To fix this problem, you need a corrected $f 77$ script. If you can edit the script yourself, change the last 3 lines from

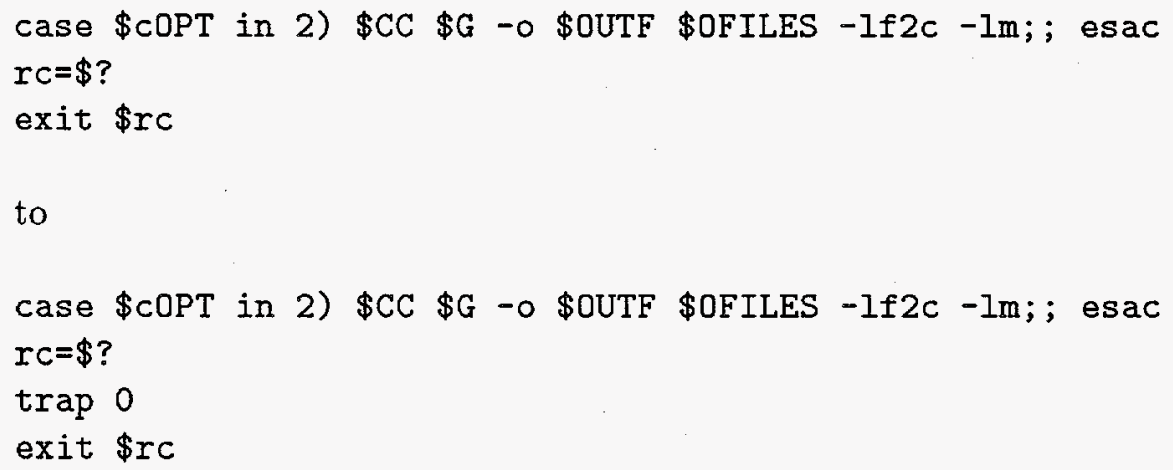

\subsection{Problems Building mpich}

\subsubsection{General}

1. Q: When running make on mpich, I get this error:

ar: write error: No such file or directory

*** Error code 1

I've looked, and all the files are accessible and have the proper permissions.

A: Check the amount of space in /tmp. This error is sometimes generated when there is insufficient space in /tmp to copy the archive (this is a step that ar takes when updating a library). The command df /tmp will show you how much space is available. Try to ensure that at least twice the size of the library is available. 
2. Q: When running make on mpich, I get errors when executing ranlib.

A: Many systems implement ranlib with the ar command, and they use the /tmp directory by default because it seems obvious that using / tmp would be faster (/tmp is often a local disk). Unfortunately, a large number of systems have ridiculously small /tmp partitions, and making any use of /tmp is very risky. In some cases, the ar commands used by mpich will succeed because they use the I option-this forces ar to use the local directory instead of / tmp. The ranlib command, on the other hand, may use /tmp and cannot be fixed.

In some cases, you will find that the ranlib command is unnecessary. In these cases, you can reconfigure with -noranlib. If you must use ranlib, either reduce the space used in / tmp or increase the size of the /tmp partition (your system administrator will need to do this). There should be at least 20-30 MBytes free in /tmp.

\subsubsection{Workstation Networks}

1. Q: When building mpich, the make fails with errors like this:

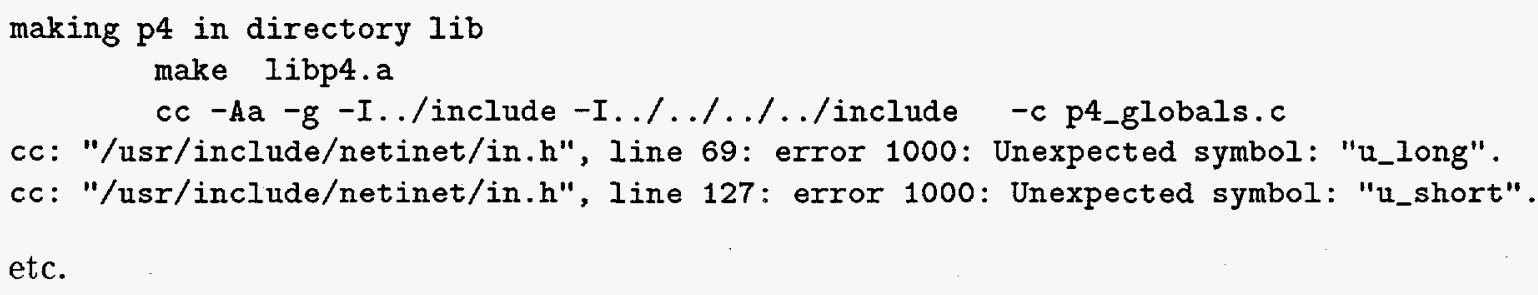

A: Check to see whether $c c$ is aliased (in $\mathrm{C}$ shell, do alias $c c$ ). If it is, either unalias it or set the environment variable CC to the full path for the compiler. To get the full path, do

unalias CC

setenv CC 'which $\mathrm{CC}^{\text {" }}$

and then reconfigure.

2. Q: When building the ch_p4 device, I get errors of the form

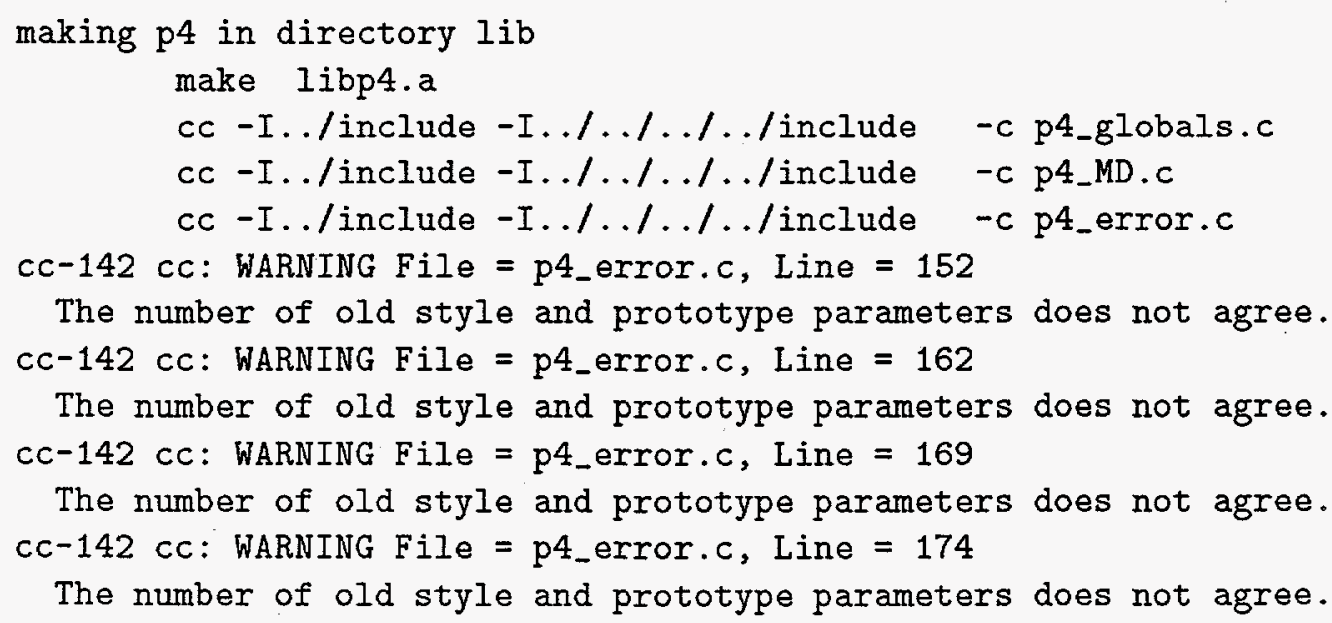

The number of old style and prototype parameters does not agree. cc-142 cc: WARNING File $=$ p4_error.c, Line $=162$

The number of old style and prototype parameters does not agree. $c c-142 \mathrm{cc}$ : WARNING File $=$ p4_error. $c$, Line $=169$

The number of old style and prototype parameters does not agree. cc-142 cc: WARNING File $=$ p4_error.c, Line $=174$

The number of old style and prototype parameters does not agree.

A: These have to do with declarations for a signal handler and can be ignored. 


\subsubsection{Cray T3D}

1. Q: When linking I get

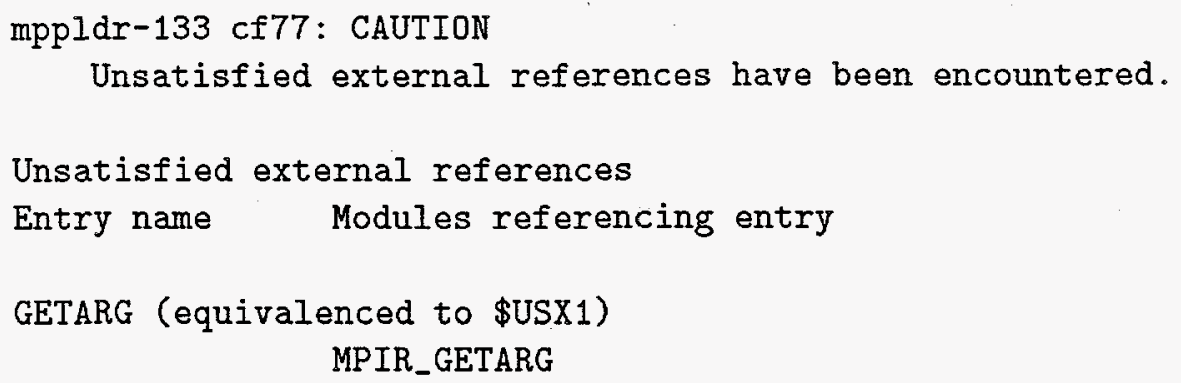

A: You may have specified the Fortran compiler with the $-f c$ argument to configure. The mpich Fortran implemenation of MPI uses a common Fortran extension, GETARG, to get the command line arguments. Most Fortran runtime systems support this, but Cray uses call pxfgetarg(i,s,len(s),ierr) instead. You can change the file src/env/farg.f manually to call the correct routine (but note that configure builds a new $f$ arg. $f$ from $f a r g . f$. in each time that it is run).

\subsubsection{Intel i860}

1. Q: The link test fails on an Intel i860 with

icc -o overtake overtake.o test.o -L/mpich/lib/intelnx/ -Impi -Inode /usr/ipsc/XDEV/i860/bin/Id860: Error: undefined symbol '_MPI_Keyval_create' /usr/ipsc/XDEV/i860/bin/ld860: Fatal: no output file created

A: You are probably building mpich on an old 386 running System $V$ release 2. This version of Unix has very severe limitations on the length of filenames (more severe than we are willing to cater to). The specific problem here is that the name of the file mpich/src/context/keyval_create.c is too long for this system and was not properly archived. You best bet is to build mpich on a different, more modern system (for example, a Sun running SunOS or Solaris).

\subsubsection{Intel Paragon}

1. Q: I got the following messages when I tried to build on the Paragon:

PGC-W-0115-Duplicate standard type (init.C: 576)

PGC/Paragon Paragon Rel R5.0: compilation completed with warnings PGC-W-0115-Duplicate standard type (init.c: 576)

PGC/Paragon Paragon Rel R5.0: compilation completed with warnings

A: This is because the compiler doesn't handle long long int but doesn't reject it either. It causes no harm. 


\subsubsection{SGI}

1. Q: The build on an SGI Power Challenge fails with

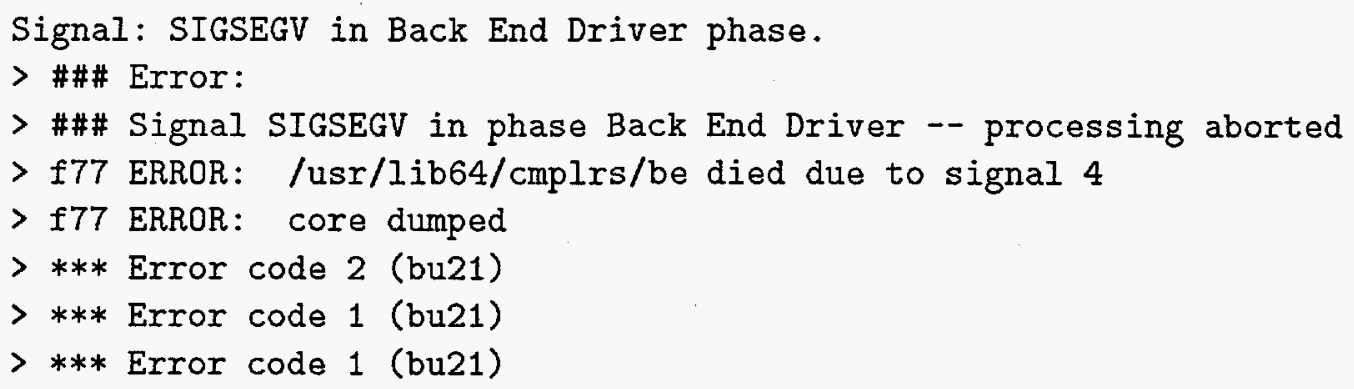

A: Our information is that setting the environment variable SGI_CC to -ansi will fix this problem.

2. Q: The build on an SGI with architecture IRIXN32 fails with

cc: Warning: - c should not be used with ucode $-03-032$ on a single file; use -jinstead to get inter-module optimization.

A: Amazingly, the standard $-c$ option is not valid for the SGI compilers when both -03 and $-n 32$ are specified. This is a feature of the SGI compiler, and there is no way to work around this for mpich (other than a massive and non-portable rewrite of all the Makefiles). Your only option is to not use the -03 option.

\subsubsection{LINUX}

1. Q: The link test failed on LINUX 3.0 with

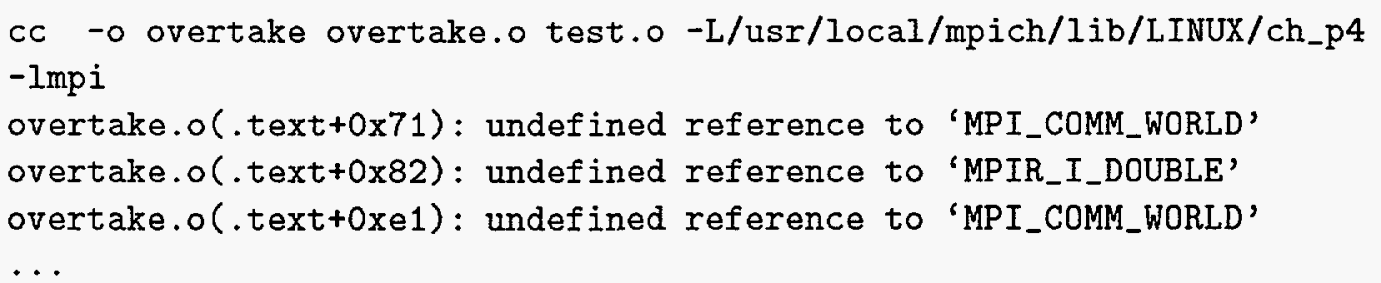

A: We have been informed that there is a error in the $f 77$ script in some versions of LINUX which causes this problem. Try either getting a patch for the $f 77$ script or reconfiguring with -nof 77 .

\subsubsection{IBM SP2}

1. Q: Linking fails on an IBM SP2 for the ch_mpl device.

A: You may have a version of the IBM MPL/POE software that already includes MPI. If that is the case, we recommend that you use that version. If you need to use MPICH, configure with -mpilibname=mpich and rebuild MPICH. 
2. Q: When trying to link on an IBM SPx, I get the message from mpirun:

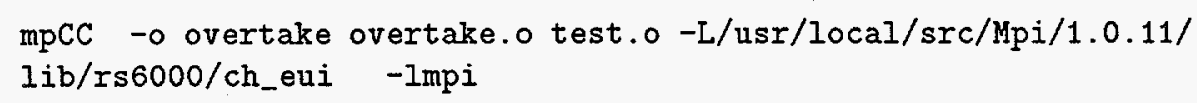

ld: 0711-317 ERROR: Undefined symbol: .mp_main

ld: 0711-317 ERROR: Undefined symbol: .mp_environ

ld: 0711-317 ERROR: Undefined symbol: .mpc_bsend

...

A: Your IBM implementation does not seem to contain the MPL routines that mpich uses to implement MPI. Your system may contain the IBM version of MPI; you should use that instead.

We have been discussing with IBM a way to provide mpich on SP systems that also have the IBM MPI; unfortunately, we have not been able to get the information that we need.

In the interim, you can do the following:

Change the use of a library path (-L/usr/local/...), followed by -1mpi, with an explicit file name. For example, instead of

-L/usr/local/src/Mpi/1.0.11/1ib/rs6000/ch_eui -lmpi

do

/usr/local/src/Mpi/1.0.11/lib/rs6000/ch_eui/libmpi.a

This change needs to be made in the Makefiles and in the scripts such as mpicc, mpif77, and mpicc.

\subsubsection{DEC ULTRIX}

1. Q: When trying to build, the make aborts early during the cleaning phase:

amon:MPICH/mpich>make clean

/bin/rm -f*.o*- nupshot

*** Error code 1

A: This is a bug in the shell support on some DEC ULTRIX systems. You may be able to work around this with

setenv PROG_ENV SYSTEM_FIVE

Configuring with -make=s5make may also work. 


\subsection{Problems in Testing}

The MPICH test suite, in examples/test, performs a fairly complete test of an MPI implementation. If there is an error, it usually indicates a problem with the implementation of MPI; if you encounter such a problem, please report it to mpi-bugs@mcs.anl.gov. However, there are a few exceptions that are described here.

1. Q: The test pt2pt/structf fails with

0 - Error in MPI_ADDRESS : Invalid argument: Address of location given to MPI_ADDRESS does not fit in Fortran integer

[0] Aborting program!

A: This is not an error; it is a gap in the MPI definition. This indicates that Fortran integers are not large enough to hold an address. This does indicate that MPI programs written in Fortran should not use the MPI_Address function on this system.

2. Q: The test env/timers fails with

Timer around sleep(1) did not give 1 second; gave 0.399949

A: The low-level software that mpich uses probably makes use of the SIGALRM signal, thus denying it to the user's program. This is not an error (the standard permits systems to make use of any signals) though it is unfortunate.

One system known to use SIGALAM is the IBM MPL/POE (device ch_mpl) software for using the High Performance Switch in the IBM SPx parallel computers.

\section{Acknowledgments}

The work described in this report has benefited from conversations with and use by a large number of people. We also thank those that have helped in the implementation of MPICH, particularly Patrick Bridges and Edward Karrels. Particular thanks goes to Nathan Doss and Anthony Skjellum for valuable help in the implementation and development of mpich. 


\section{References}

[1] Ralph Butler and Ewing Lusk. User's guide to the p4 parallel programming system. Technical Report ANL-92/17, Argonne National Laboratory, October 1992.

[2] Ralph Butler and Ewing Lusk. Monitors, messages, and clusters: The p4 parallel programming system. Parallel Computing, 20:547-564, April 1994. (Also Argonne National Laboratory, Mathematics and Computer Science Division preprint MCS-P362-0493).

[3] William Gropp and Ewing Lusk. An abstract device definition to support the implementation of a high-level message-passing interface. Preprint MCS-P342-1193, Mathematics and Computer Science Division, Argonne National Laboratory, 1993.

[4] William Gropp and Ewing Lusk. Scalable Unix tools on parallel processors. In Proceedings of the Scalable High Performance Computing Conference, pages 56-62. IEEE, 1994.

[5] William Gropp and Ewing Lusk. User's guide for mpich, a portable implementation of MPI. Technical Report ANL-96/6, Argonne National Laboratory, 1996.

[6] Virginia Herrarte and Ewing Lusk. Studying parallel program behavior with upshot. Technical Report ANL-91/15, Argonne National Laboratory, Argonne, 1991.

[7] Edward Karrels and Ewing Lusk. Performance analysis of MPI programs. In Jack Dongarra and Bernard Tourancheau, editors, Proceedings of the Workshop on Environments and Tools For Parallel Scientific Computing, pages 195-200. SIAM Publications, 1994. 


\section{Distribution for ANL-96/5}

\section{Internal:}
J. M. Beumer (10)
F. Y. Fradin
W. D. Gropp (10)
E. L. Lusk (10)
G. W. Pieper
R. L. Stevens
C. L. Wilkinson
TIS File

\section{External:}

DOE-OSTI, for distribution per UC-405 (52)

ANL-E Library

ANL-W Library

Manager, Chicago Operations Office, DOE

Mathematics and Computer Science Division Review Committee:

F. Berman, University of California at LaJolla

G. Cybenko, Dartmouth College

T. DuPont, The University of Chicago

J. G. Glimm, State University of New York at Stony Brook

M. T. Heath, University of Illinois, Urbana

E. F. Infante, University of Minnesota

$K$. Kunen, University of Wisconsin at Madison

R. E. O'Malley, University of Washington

L. R. Petzold, University of Minnesota

F. Howes, Dept. of Energy - Office of Computational and Technology Research

D. Nelson, Dept. of Energy - Office of Computational and Technology Research 